\title{
Limitations on ultrafast optical switching in a semiconductor laser amplifier operating at transparency current
}

\author{
Y.-H. Kao, ${ }^{\text {a) }}$ T. J. Xia, and M. N. Islam \\ Department of Electrical Engineering and Computer Science, University of Michigan, Ann Arbor, \\ Michigan 48109 \\ G. Raybon \\ Lucent Technologies, Holmdel, New Jersey 07733
}

(Received 1 March 1999; accepted for publication 31 July 1999)

\begin{abstract}
Ultrafast optical switching in a semiconductor laser amplifier (SLA) at transparency current is studied under a strong pump condition. The switch configuration is a nonlinear optical loop mirror with a SLA as the nonlinear element. We demonstrate optical switching with 2 ps recovery time and $60 \%$ nonlinear transmission at switching energy of $9 \mathrm{pJ}$. We find that the transparency current is pump power dependent and that the transparency current is different for uniform 7-bit input control pulses at $100 \mathrm{~Gb} / \mathrm{s}$. We believe these two outcomes are due to significant carrier generation via two photon absorption (TPA) at high pump intensity. To verify our hypothesis, we modify coupled propagation equations by including the carrier generation due to the TPA and solve the equations numerically. Good agreement between the experimental and simulation results is obtained. We conclude that to achieve complete pattern-independent $100 \mathrm{~Gb} / \mathrm{s}$ optical switching using a SLA at transparency current, we have to avoid TPA or use the SLA with a transit time shorter than the control pulse width. (C) 1999 American Institute of Physics. [S0021-8979(99)06221-0]
\end{abstract}

\section{INTRODUCTION}

In a high-speed optical time division multiplexed (OTDM) network, all-optical switches can perform ultrafast signal processing without optoelectronic conversion. Semiconductor laser amplifiers (SLAs) have been considered as promising optical switching devices because of their compactness, their ability to be integrated, and their environmental stability. The main challenge for SLA based devices is the existence of a slow nonlinearity (recovery time $\approx \mathrm{ns}$ ). Several approaches have been developed to solve this slow recovery problem. One is to enhance the recovery rate by injecting a strong continuous wave (cw) "holding beam" into the SLA. ${ }^{1}$ A $\pi$ phase change that recovers within $12.5 \mathrm{ps}$ with a $20 \mathrm{~mW}$ cw holding beam has been demonstrated. ${ }^{2}$ Another approach is to design a switch configuration such that the phase shifts due to the slow nonlinearity are about the same as those for two arms of the interferometer. ${ }^{3,4} \mathrm{Al}-$ though this approach cannot avoid amplitude modulation due to the slow gain saturation effect, $100 \mathrm{~Gb} / \mathrm{s}$ logic operations have been demonstrated. ${ }^{4}$

Besides these two approaches, recent femtosecond pump-probe measurements revealed a large $\left(\approx 10^{-12} \mathrm{~cm}^{2} / \mathrm{W}\right)$ and fast recover $(\approx \mathrm{ps})$ nonlinear refractive index in SLAs biased at transparency current where no net slow inter band transitions were induced by the pump pulses. ${ }^{5}$ At this transparency current condition, both nonlinear index and nonlinear gain recover in picoseconds, ${ }^{5}$ therefore, ultrafast and pattern-independent switching can be achieved. Nonlinear switching in directional couplers ${ }^{6}$ and a

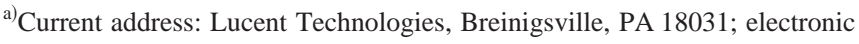
mail: yuanhuakao@lucent.com
}

$10 \mathrm{~Gb} / \mathrm{s}$ AND gate in an ultrafast nonlinear interferometer ${ }^{7}$ using SLAs biased at transparency current have been demonstrated. We have also reported an AND gate with a 2 ps switching recovery time in a loop mirror at transparency current. ${ }^{8}$ We observed that at high control pulse power $(5 \mathrm{~W}$ peak power) nonlinear absorption was not negligible and the transparency current was power dependent. ${ }^{8,9}$ To achieve maximum switching output, high control pulse power is required; therefore, the effect of two photon absorption (TPA) on switching performance needs to be understood. The TPA limitation to optical switching has been studied with regard to TPA induced loss. ${ }^{10,11}$ The TPA effect on carrier heating ${ }^{12}$ and picosecond pulse propagation ${ }^{13,14}$ has also been analyzed. However, the effect of TPA on optical switching the transparency condition due to TPA generated carriers and TPA induced control pulse distortion has not been examined.

In this article, we study optical switching in a SLA biased at transparency current under strong control pulse power. We begin in Sec. II by performing pump-probe measurements to determine the transparency current under strong pump conditions. In Sec. III, we characterize the switching performance at transparency current. We first use a strong single bit control pulse. In this case, fast recovered switching is obtained at the transparency condition. To characterize the switching performance at $100 \mathrm{~Gb} / \mathrm{s}$, we generate a 7-bit " 1011001 " packet at a bit rate of $100 \mathrm{~Gb} / \mathrm{s}$ as the control pulse. The peak power for each control bit is higher than 6 $\mathrm{W}$, which generates a significant number of carriers through two photon absorption. In this case, no transparency condition can be obtained for every control bit and no fast switching can be achieved for every bit, although each control bit 
has similar input peak power. We attribute this to a combination of TPA carriers and control pulse depletion in the SLA. To verify our hypothesis, we numerically solve coupled propagation equations including gain depletion, carrier heating, and carrier generation due to TPA. Both rapid gain and phase dynamics are included in our model to simulate carrier dynamics as well as pulse propagation. We obtain good agreement between the simulation results and experimental results for the pump-probe measurements. We find that the TPA carrier generation term is necessary to explain why transparency currents are pump power dependent. We further use this simulation to show that carrier dynamics induced by the pump are different at different positions of the SLA because of pump depletion caused by the TPA. Therefore, carrier densities in the SLA are different at different propagation distances and the transparency condition cannot be satisfied at every position of the SLA. Although one can adjust the current for the first control pulse such that the SLA, on average, is at the transparency condition, the carrier distribution in the SLA has been changed to a different condition and one will need to readjust the current to achieve a transparency condition for the second control pulse. Therefore, transparency conditions become different for the first and subsequent control pulses before SLA carriers reach steady state.

\section{CROSS-POLARIZED PUMP-PROBE EXPERIMENTS}

To study the transparency condition at high pump power, we perform pump-probe experiments. The pump and probe pulses are generated from a passively mode-locked erbium-doped fiber laser (EDFL) with a pulse width of $0.8 \mathrm{ps}$ at $1541 \mathrm{~nm}$. The repetition rate is about $20 \mathrm{MHz}$. The pulses are split into the pump and the probe via an acousto-optic modulator $(\mathrm{AOM})$ and the pump pulses are chopped by a chopper. A variable delay stage is used to adjust the time delay between the pump and the probe pulses. The pump and probe pulses are combined by a 50/50 coupler and coupled into (out of) the SLA by a pair of lensed fiber (LF) with coupling efficiency of $10 \%$. Polarization controllers (PCs) are used to adjust the pump polarization to the (TE) polarization [along the multiple quantum well MQW layers] and the probe polarization to TM. The SLA is a $470 \mu \mathrm{m}$ long InGaAsP MQW with antireflection coating on both sides. The pump pulses are filtered out by a polarizer at the output of the SLA and the pump-induced probe transmission change is measured by a slow detector and a lock-in amplifier.

Figure 1 shows the pump-probe results at pump energy of $5 \mathrm{pJ}$ (peak power of $6.25 \mathrm{~W}$ ) and $6 \mathrm{pJ}$ (peak power of 7.5 $\mathrm{W})$. The delay is defined as negative when the pump is behind the probe. At $5 \mathrm{pJ}$, the probe transmission to its original level a few picoseconds after the pump passes the SLA. This indicates that the carrier density in the SLA is the same before and after the pump pulses pass through the SLA and that the SLA is at the transparency condition. However, when we increase the pump energy to $6 \mathrm{pJ}$, the probe transmission is higher after the pump passes the SLA. We believe that this power dependent transparency current is due to the effect of TPA. The high pump power induces a significant amount of

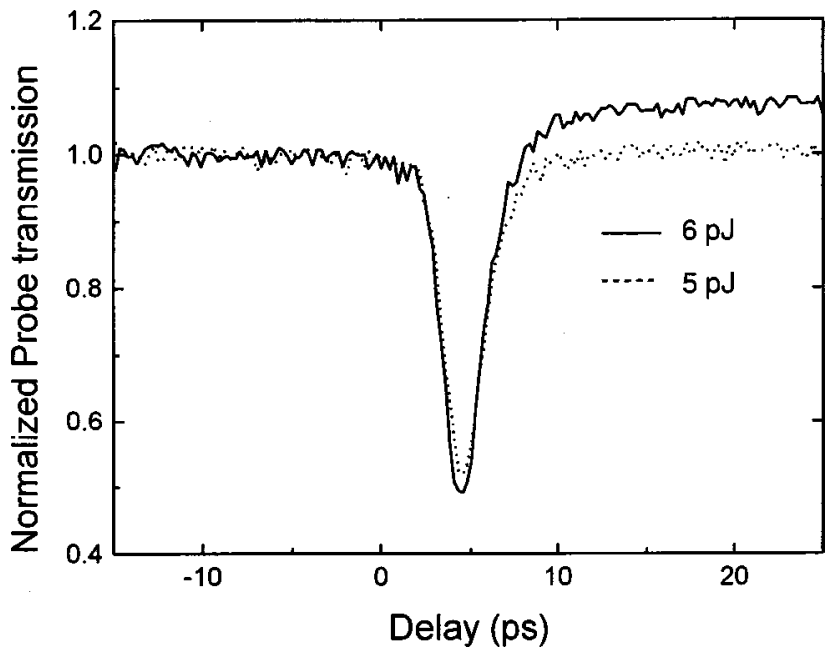

FIG. 1. Normalized probe transmission as a function of pump-probe delay at pump power of 5 (dotted line) and $6 \mathrm{pJ}$ (solid line). The bias current is 36 $\mathrm{mA}$.

TPA carriers that are pump power dependent. The pump pulse generates more carriers from TPA at $6 \mathrm{pJ}$, so it requires a higher current to reach the transparency condition in the SLA. Figure 2 illustrates the transparency current as a function of the input pump energy. This power dependent behavior of the transparency current was also observed by Kennedy et al. ${ }^{9}$ using longer (14-30 ps) pulses.

\section{EXPERIMENTAL RESULTS FOR ULTRAFAST SWITCHING IN SLAS}

The experimental setup for an ultrafast AND gate is illustrated in Fig. 3. The control pulse is brought into the loop mirror by a $3 \mathrm{~dB}$ coupler to induce nonlinearity in the SLA, and the signal pulse is split into two counterpropagating signals by a $3 \mathrm{~dB}$ coupler in the loop. The two signals interfere at the coupler after they experience a phase shift in the SLA and result in switching. A variable air gap is used to adjust

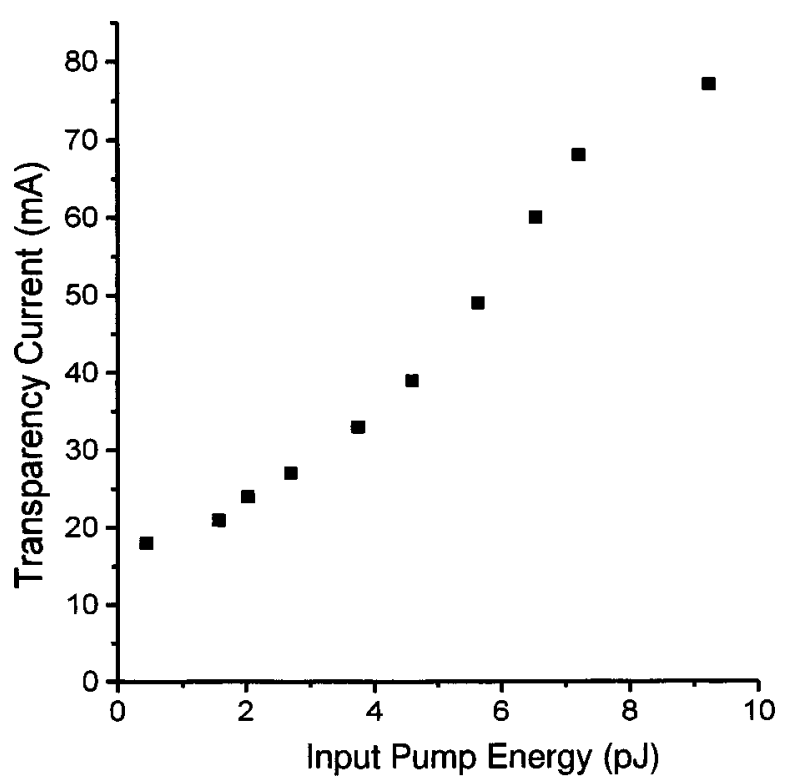

FIG. 2. Transparency current as a function of the input pump energy. 


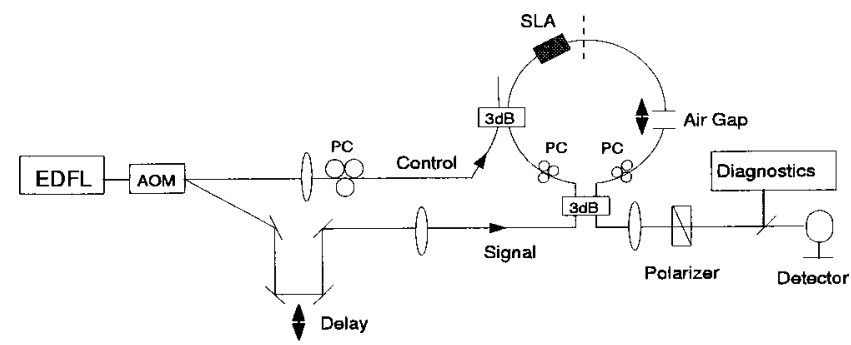

FIG. 3. Experimental setup for the SLA switch. EDFL—erbium-doped fiber laser, $\mathrm{AOM}$-acousto-optic modulator, and $\mathrm{PC}$-polarization controller.

the SLA position in the loop. Again, the control is TE polarized and the signal is TM polarized before being coupled into the SLA. The control pulses are filtered out by a polarizer at the output of the loop and the signal pulses that are switched out are either measured by a slow detector or diagnosed by an autocorrelator and a spectrometer.

To demonstrate ultrafast optical switching at transparency current, we measure the time-averaged signal transmission at the loop output as a function of the time delay between the control and the signal in the gain, transparency, and absorption regimes (Fig. 4). ${ }^{8}$ The loop is adjusted to minimize the signal transmission in the absence of the control. The control pulse energy coupled into the SLA is about $3 \mathrm{pJ}$ ( $3.75 \mathrm{~W}$ peak power), which is about 10 times the signal energy. In the gain regime, the timing window is $20 \mathrm{ps,}$ which is determined by the offset of the SLA from the center of the loop. The structure in the timing window illustrates

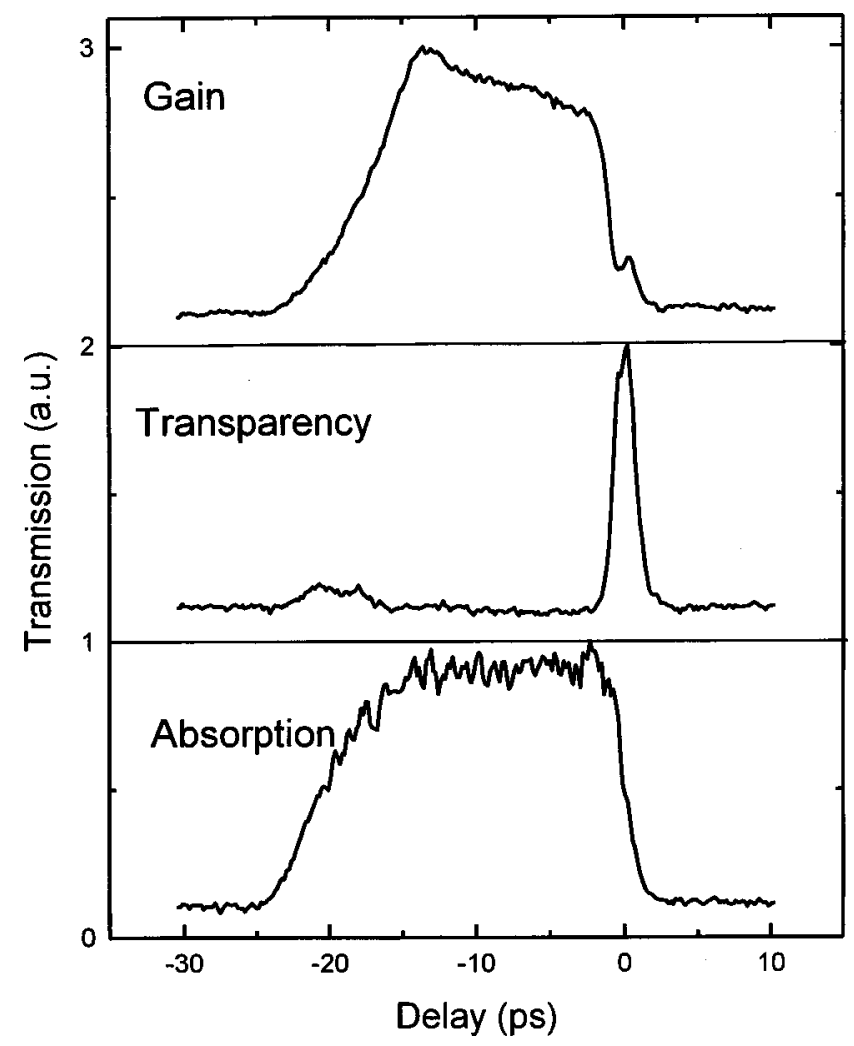

FIG. 4. Time-averaged signal transmission at the loop output as a function of time delay between signal and control pulses at different currents. The transparency current is $31 \mathrm{~mA}$.

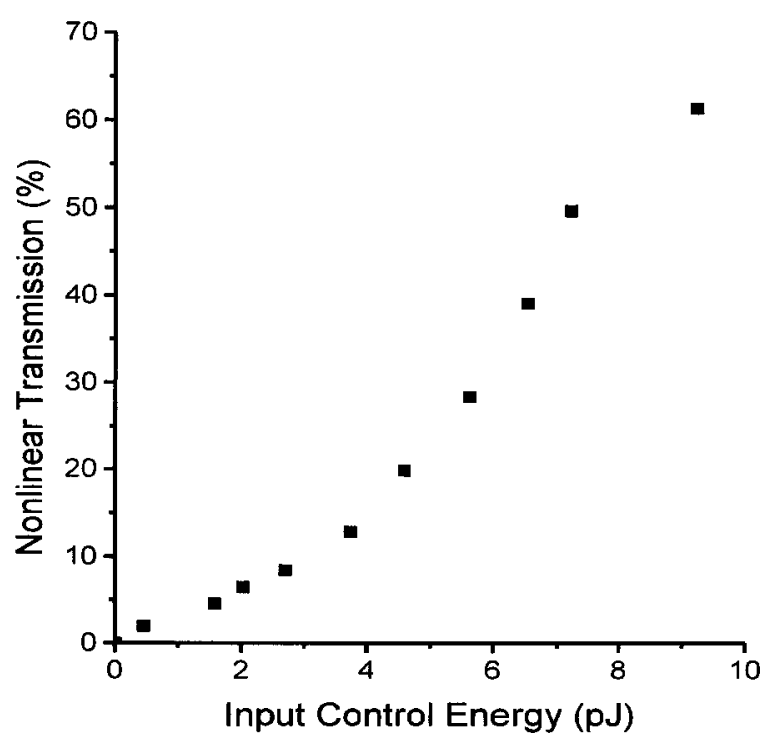

FIG. 5. Ratio between the nonlinear transmission (with the control pulse) and the linear transmission (without the control pulse) as a function of the input control energy.

different recovery times of the fast and slow nonlinearities. In the absorption regime, the nonlinear switching is dominated by the slow nonlinearity and the timing window is 20 ps. At transparency current, the timing window reduces to 2 ps, which is the convolution of the signal, control, and the ultrafast carrier recovery time. The small timing window at a delay of -20 ps occurs because the control pulse overlaps the counterpropagating signal pulse in the SLA and switches out the counterpropagating signal. The counterpropagating signal that is switched out is much weaker than the copropagating signal that is switched out due to a shorter interaction length and control pulse depletion due to the TPA. This sharp timing window indicates that the SLA can perform ultrafast switching in $2 \mathrm{ps}$ at transparency current. Therefore, by adjusting the current to the transparency condition, we should be able to achieve $100 \mathrm{~Gb} / \mathrm{s}$ optical switching.

We further characterize the gate performance in terms of maximum nonlinear transmission, switching energy, and output pulse quality. The control pulses are amplified by an erbium-doped fiber amplifier (EDFA) to achieve higher switching output. Figure 5 shows the ratio between nonlinear transmission (with control pulse) and linear transmission of the gate as a function of the input control energy coupled into the SLA. The linear transmission is obtained by adjusting the loop to maximize the signal transmission without the control pulse. Note that the current needs to be readjusted to the transparency condition at each input control energy (Fig. 2 ). The maximum nonlinear transmission is about $60 \%$ at input control energy of about $9 \mathrm{pJ}$. The maximum nonlinear transmission is limited to $60 \%$ because the control pulse is distorted by TPA at high energy. ${ }^{14,15}$ Although the control pulse is distorted, we find that the signal pulse is not distorted significantly because of its low power. Figure 6 shows the autocorrelation and the spectrum of the input signal pulse (dashed line) and switched-out signal pulse (solid line). By comparing the dashed and solid lines in Fig. 6, we conclude 

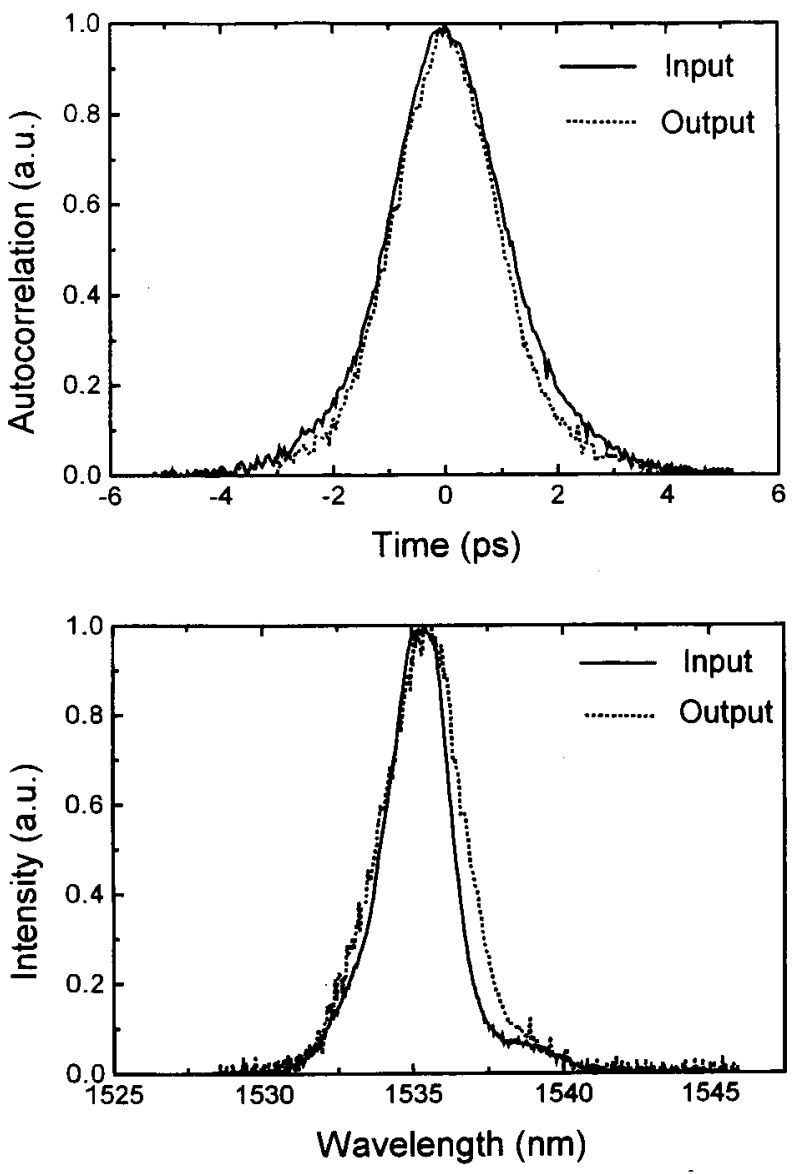

FIG. 6. Auto-correlation (top) and optical spectrum (bottom) of the signal pulse at the loop output with (dashed line) and without (solid line) the SLA.

that the signal pulse distortion from this gate is insignificant and it can be amplified and used to drive another gate.

So far we have demonstrated a fast recovered switching characteristic using the SLA at transparency current with 2 ps pulses at a slow repetition rate $(20 \mathrm{MHz})$. To emulate the switching performance at $100 \mathrm{~Gb} / \mathrm{s}$, we use a 7-bit $100 \mathrm{~Gb} / \mathrm{s}$ packet 1011001 as the control pulses. This packet is formed by $1 \times 4$ and $4 \times 1$ couplers and amplified by an EDFA with a pulse width of $1.5 \mathrm{ps}$ and pulse energy of $9 \mathrm{pJ} /$ pulse $(6 \mathrm{~W}$ peak power). The signal pulse is still a single bit direct from the EDFL. The SLA offset is adjusted to be about 8 ps from the loop center. Figure 7 shows the input 7-bit control pulses and signal transmissions at the switch output as a function of the delay between the control and signal. The 7-bit control pulses have uniform input power, but only the first pulse has the fast recovered timing window. The timing windows from the other three control pulses consist of a big window from the slow nonlinearity and a narrow window from the fast nonlinearity. The presence of the big timing window implies that there is switching output when the control does not overlap the signal and the SLA is not at the transparency condition. We can also adjust the current such that only the second bit is at the transparency condition, but we cannot find any current which reaches the transparency condition for every control bit. Figure 8 shows the pump-probe results corresponding to Fig. 7. Note that the probe transmission recovers to the original level for the first pump pulse, but not for the

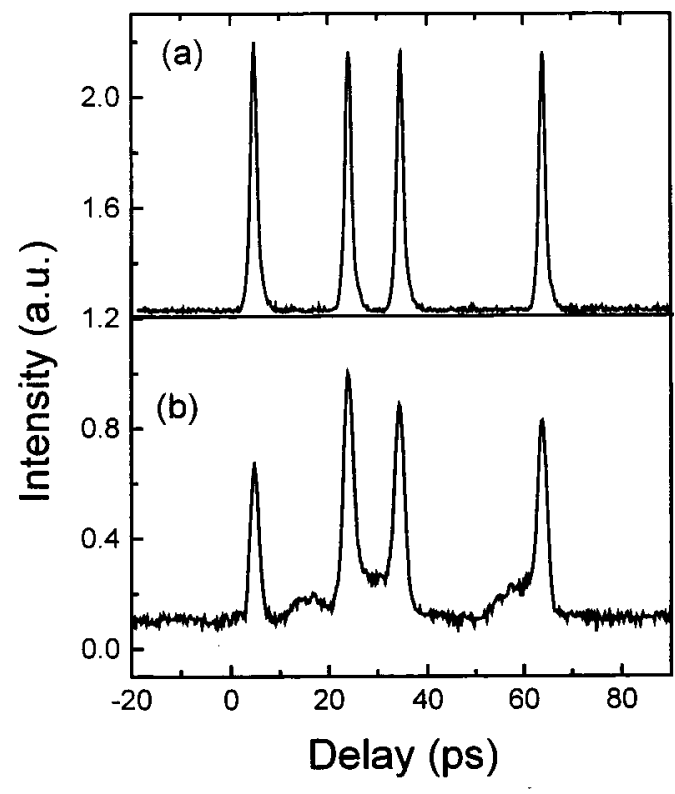

FIG. 7. (a) Cross correlation of the input 7-bit 1011001 control pulses. (b) Time-averaged signal transmission at the loop output as a function of time delay between signal and control pulses at bias current of $30 \mathrm{~mA}$.

rest of the pump pulses. This further proves that the SLA is at transparency only for the first pump pulse.

The 7-bit switching results indicate that the transparency conditions are different for different control bits, although they have the same input power. We attribute this to the effect of TPA and pump depletion. Because the control pulse width (1.5 ps) is shorter than the SLA transit time (5 ps), the control peak power decreases due to the TPA as it propagates in the SLA. Therefore, the TPA generated carrier number decreases along the propagation and it requires different currents to reach the transparency condition at different positions of the SLA. For the first control pulse, we can find a current such that on average the SLA is at the transparency point. In other words, the beginning part of the SLA and the latter part of the SLA are in either the gain or the absorption

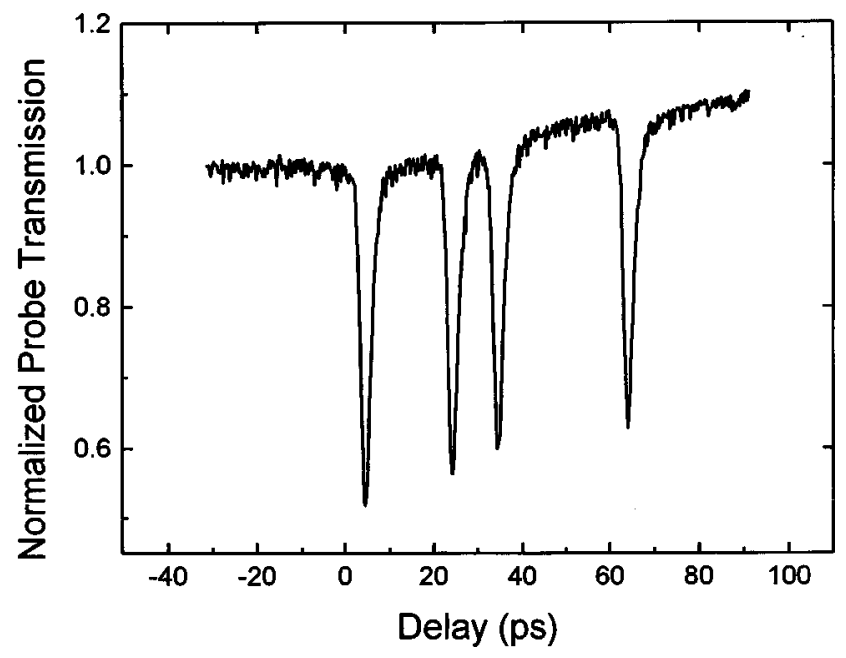

FIG. 8. Normalized probe transmission as a function of time delay between the 7-bit 1011001 pump pulses and the probe pulse at bias current of 30 $\mathrm{mA}$. 
regime, but the SLA is at transparency on average. However, when the second control pulse enters the SLA, it experiences different carrier densities at different positions of the SLA and experiences different initial conditions compared to those of the first control pulse. Therefore, it requires different currents to achieve the transparency condition on average, and we cannot find a transparency current for $100 \mathrm{~Gb} / \mathrm{s}$ pulses before the SLA reaches its steady state.

\section{COUPLED PROPAGATION EQUATIONS}

To understand the intensity dependent transparency current and switching results at $100 \mathrm{~Gb} / \mathrm{s}$, we modify the coupled propagation equations in Refs. 13 and 15 by including the carrier generation due to the TPA effect. The resultant coupled propagation equations for the pump and the probe pulses are

$$
\begin{aligned}
\frac{\partial}{\partial z} S(t, z)= & \left(-\frac{\beta}{2 A_{\mathrm{eff}}}+i \frac{\omega_{0}}{c A_{\mathrm{eff}}} n_{2}\right)|S(t, z)|^{2} S(t, z) \\
& +\left[\frac{1}{2} g_{N}\left(t, \omega_{0}\right)\left(1+i \alpha_{N}\right)\right] S(t, z) \\
& +\frac{1}{2} \Delta g_{\mathrm{CH}}\left(t, \omega_{0}\right)\left(1+i \alpha_{\mathrm{CH}}\right) S(t, z) \\
& +\frac{1}{2} \Delta g_{\mathrm{TPA}}\left(t, \omega_{0}\right)\left(1+i \alpha_{\mathrm{TPA}}\right) S(t, z)
\end{aligned}
$$

and

$$
\begin{aligned}
\frac{\partial}{\partial z} p(t, z)= & 2\left(-\frac{\beta}{2 A_{\mathrm{eff}}}+\frac{i}{3} \frac{\omega_{0}}{c A_{\mathrm{eff}}} n_{2}\right)|S(t, z)|^{2} p(t, z) \\
& +\left[\frac{1}{2} g_{N}\left(t, \omega_{0}\right)\left(1+i \alpha_{N}\right)\right] p(t, z) \\
& +\frac{1}{2} \Delta g_{\mathrm{CH}}\left(t, \omega_{0}\right)\left(1+i \alpha_{\mathrm{CH}}\right) p(t, z) \\
& +\frac{1}{2} \Delta g_{\mathrm{TPA}}\left(t, \omega_{0}\right)\left(1+i \alpha_{\mathrm{TPA}}\right) p(t, z),
\end{aligned}
$$

where $S(t, z)$ is the slowly varying envelope function of the electric field for the pump pulse and $p(t, z)$ is that for the probe pulse. The terms on the right-hand side of both equations are the TPA, the corresponding instantaneous nonlinear refractive index $n_{2}$, the gain saturation from carrier depletion $g_{N}$, the associated index change through the linewidth enhancement factor $\alpha_{N}$, an additional gain reduction from the carrier heating effect $\Delta g_{\mathrm{CH}}$, the associated index change through $\alpha_{\mathrm{CH}}$, the gain increase from the carrier generated by TPA $\Delta g_{\text {TPA }}$, and the associated index change through $\alpha_{\text {TPA }}$. Neither the group velocity dispersion (GVD) due to the background index nor spectral hole burning are included in our propagation equation because they are not significant for the 0.8 ps pulses. ${ }^{5,16} \mathrm{We}$ also neglect the gain dispersion terms because the pulse distortions due to the gain dispersion do not affect the overall carrier density significantly. The $A_{\text {eff }}$ is the effective area $w d / \Gamma$, where $w d$ is the cross section of the active region and $\Gamma$ is the confinement factor.

Since the pulse width is much shorter than the carrier lifetime $(\approx$ ns), the gain changes due to the carrier depletion $g_{N}(t)$ are given by ${ }^{17}$

$$
g_{N}(t)=g_{0} \exp \left(-\frac{1}{W_{s}} \int_{-\infty}^{t}|S(s)|^{2} d s\right),
$$

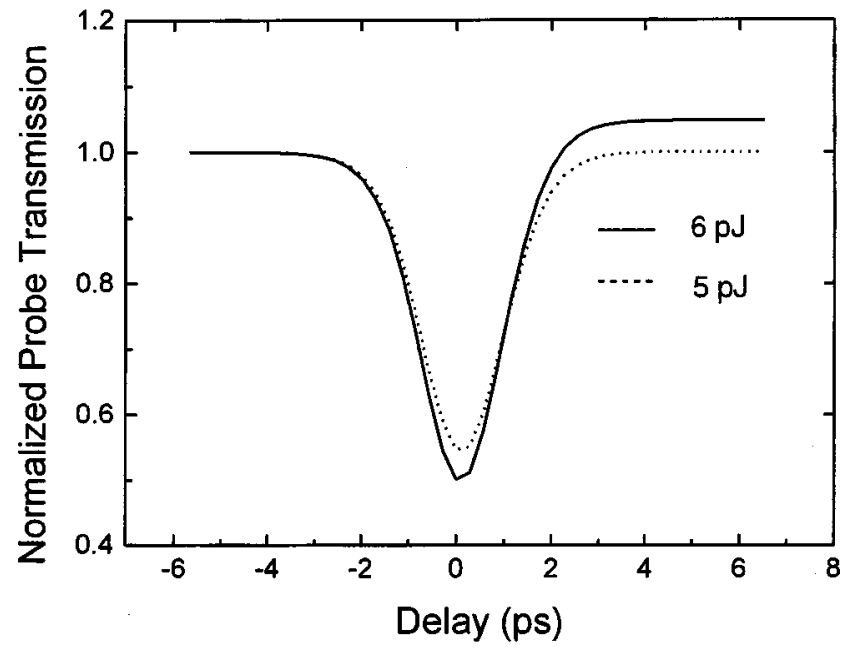

FIG. 9. Simulated probe transmission as a function of delay between the pump and the probe. The pump pulse energy is 5 (dotted curve) and $6 \mathrm{pJ}$ (solid curve).

where $g_{0}$ is the small signal gain, and $W_{s}$ is the saturation energy.

The gain reduction caused by the carrier heating effect is described as

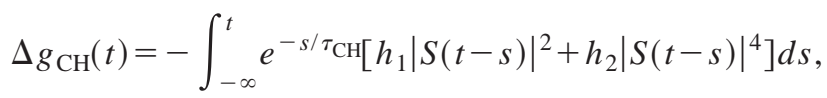

where $\tau_{\mathrm{CH}}$ is the relaxation time of the heated carrier temperature back to the lattice temperature due to carrierphonon scattering, $h_{1}$ describes the carrier heating contribution from free carrier absorption, and $h_{2}$ describes the contribution from the TPA. Because we are also interested in the carrier density change after the pump pulse propagates through the SLA, we need to consider the carriers generated by the TPA. The TPA effect first reduces the gain by heating up the carrier distribution and then increases the gain by increasing the carrier density. By introducing a third level in the rate equation, we derive the gain increase due to carrier generated by the TPA $\Delta g_{\mathrm{TPA}}(t)$ as

$$
\Delta g_{\mathrm{TPA}}(t)=\frac{1}{\tau_{\mathrm{CH}}} \int_{-\infty}^{t} d t \int_{-\infty}^{s} e^{-s / \tau_{\mathrm{CH}}} h_{2}|S(t-s)|^{4} d s .
$$

These two propagation equations describe the pulse evolution in the SLA for both pump and probe pulses due to the nonlinearities excited by the pump pulse. By solving Eq. (4.2) at different delays between the pump and the probe pulses, we can obtain the amount of probe transmission after propagating through the SLA at different delays between the pump and the probe.

\section{SIMULATION RESULTS}

We first simulate the single bit pump-probe experiments (Fig. 1) and plot the simulation results in Fig. 9. The simulation parameters are listed in Table I. At $5 \mathrm{pJ}$ (dashed curve in Fig. 9), the probe transmission recovers to the same level a few picoseconds after the pump passes the SLA. To repro- 
TABLE I. Simulation parameters.

\begin{tabular}{lcl}
\hline \hline Parameter & Value & \multicolumn{1}{c}{ Units } \\
\hline$W_{s}$ & 10 & $\mathrm{pJ}$ \\
$A_{\text {eff }}$ & 1 & $\mu \mathrm{m}^{2}$ \\
$L$ & 430 & $\mu \mathrm{m}$ \\
$n_{2}$ & $-4.5 \times 10^{-13}$ & $\mathrm{~cm}^{2} \mathrm{~W}^{-1}$ \\
$\beta$ & 60 & $\mathrm{~cm} \mathrm{GW}^{-1}$ \\
$\tau_{\mathrm{CH}}$ & 1 & $\mathrm{ps}^{-1} \mathrm{pJ}^{-1}$ \\
$h_{1}$ & 0.3 & $\mathrm{~cm}^{-2} \mathrm{fs} \mathrm{cm}^{-1} \mathrm{pJ}^{-2}$ \\
$h_{2}$ & 300 & \\
$\alpha_{N}$ & 3 & \\
$\alpha_{\mathrm{CH}}$ & 2 & \\
$\alpha_{\mathrm{TPA}}$ & 3 & \\
\hline \hline
\end{tabular}

duce the curve at $6 \mathrm{pJ}$ (solid curve in Fig. 9), we need to include the $\Delta g_{\text {TPA }}$ term in Eq. (4.2). Without this term, the probe transmission always recovers to the same level after the pump pulse passes the SLA regardless of the input pump energy. Figure 10 shows the pump-probe results for two different input pump energies at transparency conditions when the $\Delta g_{\text {TPA }}$ term is not considered. The dip is larger at higher pump energy because the TPA is stronger, but the probe transmission recovers to the same level even at higher pump energy. Therefore, we confirm that the intensity dependent transparency condition is due to the carriers generated by the TPA.

To verify that the carrier densities in the SLA are different at different propagation distances due to TPA generated carriers, we plot the pump-induced gain changes as functions of time at different segments of the SLA in Fig. 11. The resulting pump-probe measurement for Fig. 11 is shown by the dotted curve in Fig. 9, which is typically defined as the transparency condition. Although the probe pulse experiences overall the same gain changes before and after the pump passes through, Fig. 11 shows that the slow components of the gain changes induced by the pump are different at different positions of the SLA. In other words, although

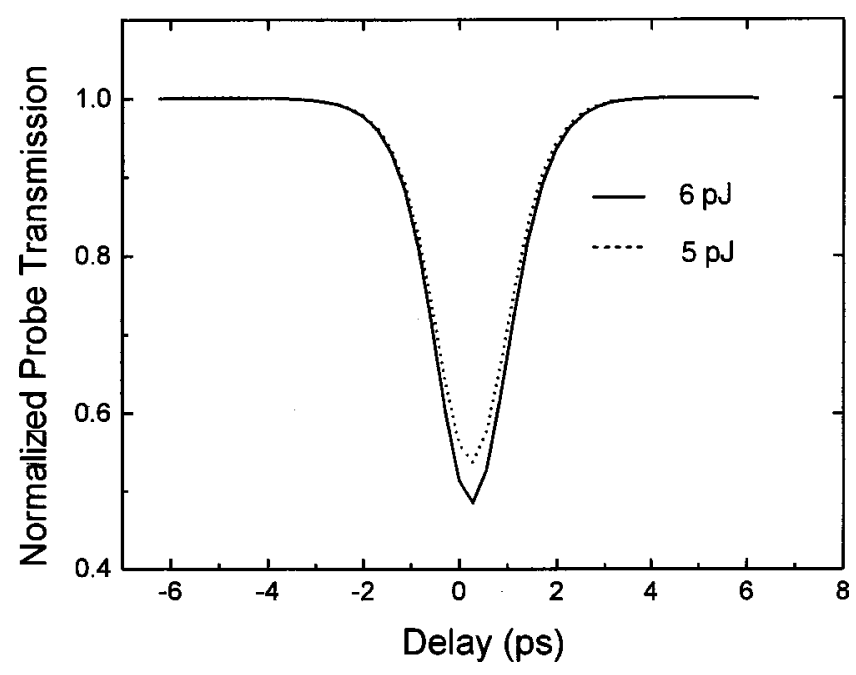

FIG. 10. Simulated probe transmission as a function of the delay between the pump and the probe when the $\Delta g_{\text {TPA }}$ term is turned off. The pump pulse energy is 5 (dotted line) and $6 \mathrm{pJ}$ (solid line).

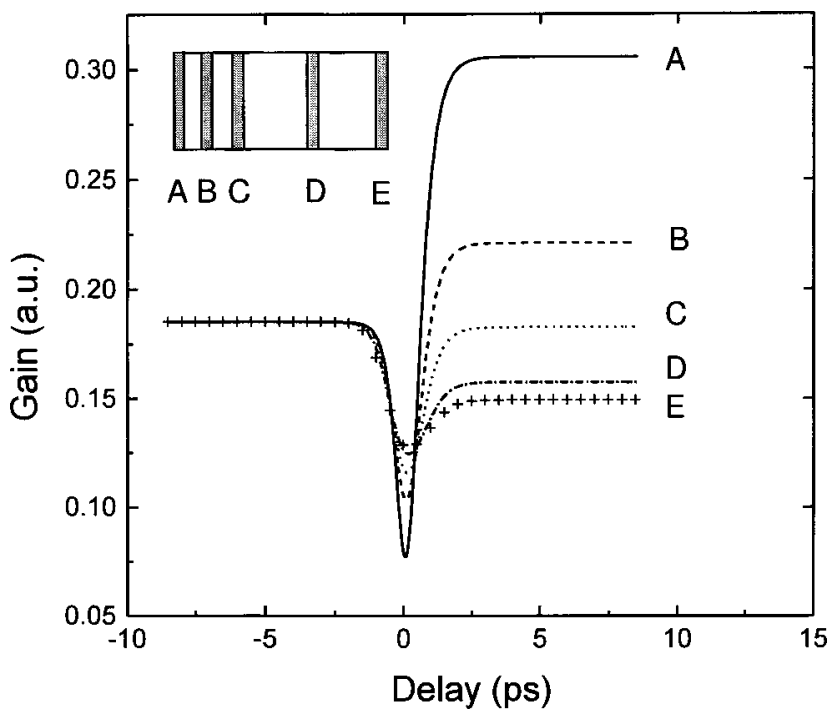

FIG. 11. Gain dynamics due to the strong pump pulse as a function of time at different positions of the SLA: $(A)=14,(B)=72,(C)=143,(D)=286$, (E) $=430 \mu \mathrm{m}$. The inset plots the segments in the SLA where the gain curves are obtained.

the pump-probe results show that the SLA is at the transparency condition, the probe does not experience the transparency condition at all positions of the SLA. The pump depletion caused by TPA results in a decrease of the TPA induced carriers along the SLA. Figure 12 illustrates the pump depletion in the SLA. For comparison, we plot the gain changes at different positions of the SLA under transparency conditions when $\Delta g_{\mathrm{TPA}}=0$ (no TPA generated carriers) (Fig. 13). We observe that the slow components of the gain changes always recover at all segments in the SLA. This shows that the whole SLA is at the transparency condition when no TPA carriers are present.

Because the slow components of the gain changes induced by the pump are different at different positions of the SLA, the second pump pulse entering the SLA 20 ps after the first pump pulse experiences different carrier density in the SLA from the first pulse. Therefore, it requires a different current to achieve the transparency condition for the second

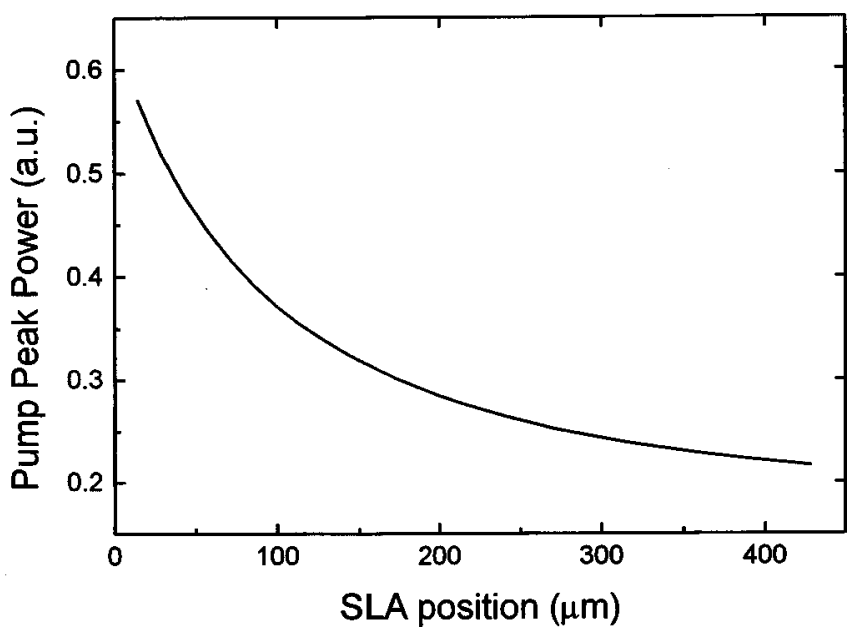

FIG. 12. Pump pulse peak power as a function of the SLA position. 


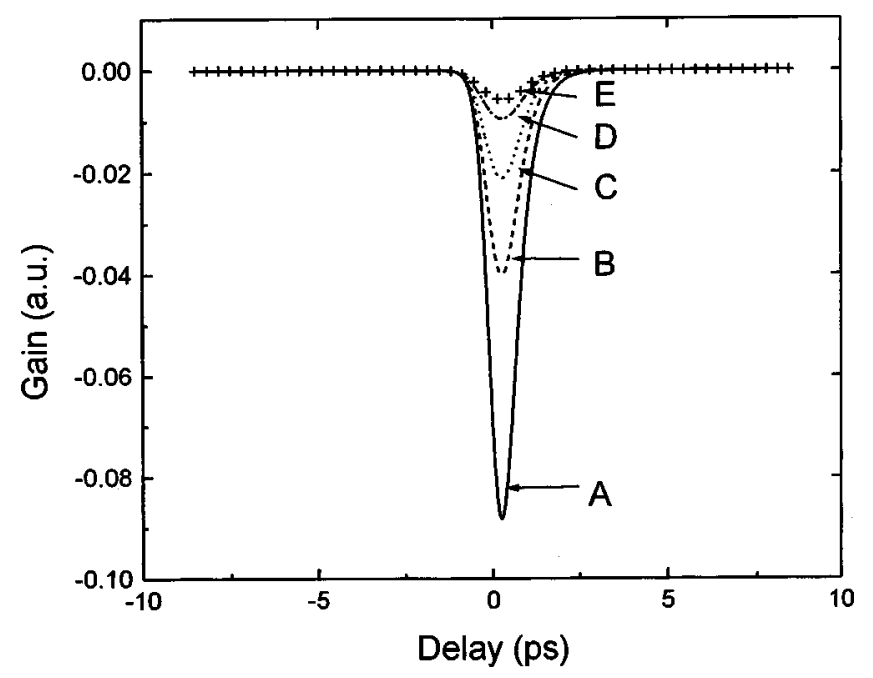

FIG. 13. Gain dynamics due to the strong pump pulse as a function of time at different positions of the SLA: $(A)=14,(B)=72,(C)=143,(D)=286$, (E) $=430 \mu \mathrm{m}$ when the $\Delta g_{\text {TPA }}$ term is turned off.

pump pulse and the transparency current will be different for the subsequent bits before the SLA reaches its steady state.

\section{DISCUSSION}

The good agreement between the experimental (Fig. 1) and simulation results (Fig. 9) verifies that TPA carriers are significant at high pump power. Although the SLA can be at a transparency condition on average in the presence of TPA carriers, the TPA carriers cause the transparency current to be pump power dependent, which makes the device less practical. Therefore, pump power should be kept low so that TPA carriers are not significant. Also, our results show that when the control pulse width is shorter than the SLA transit time and the TPA effect is significant, the SLA cannot reach the transparency condition for $100 \mathrm{~Gb} / \mathrm{s}$ pulses. Although the SLA carriers will eventually reach steady state and the transparency current will be the same for all the incoming control pulses, the switching will not be completely transparent to the control bit pattern.

One way to reach the transparency condition in the presence of significant TPA carriers is to use a control pulse width that is longer than the SLA transit time. Figure 14 plots the simulated gain changes induced by the pump at the beginning and the end of the SLA. All the parameters are the same as those in Fig. 11 except that the SLA transit time is changed to $0.1 \mathrm{ps}$ (less than one tenth of the control pulse width). We observe that the slow components recover to similar levels at both ends of the SLA even though the TPA is significant. Therefore, the whole SLA is at the same transparency condition and the transparency current is the same for all pulses. This approach may not be practical for 100 $\mathrm{Gb} / \mathrm{s}$ pulses because it would require very high switching power to obtain a $\pi$ phase shift in the SLA that is shorter than the pulse widths (typically less than $2 \mathrm{ps}$ ). Therefore, the better way to achieve $100 \mathrm{~Gb} / \mathrm{s}$ switching using SLAs at the transparency condition is to lower the switching power to

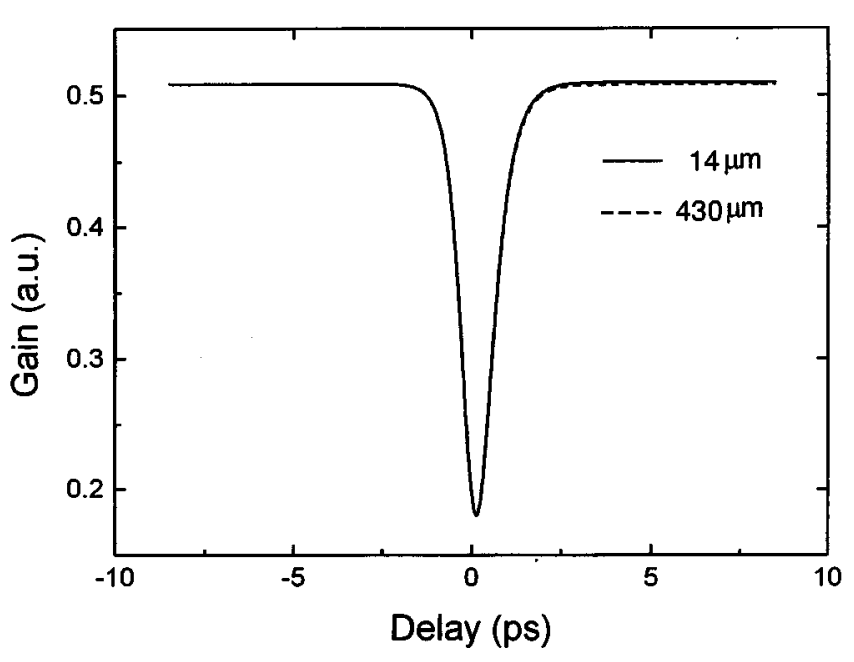

FIG. 14. Gain dynamics due to the strong pump pulse as a function of time at the beginning (solid curve) and the end (dashed curve) of the SLA. The transit time of the SLA is $0.1 \mathrm{ps}$.

avoid a strong TPA effect. One can achieve this by tuning the operating wavelength to where the nonlinear index is higher.

\section{SUMMARY}

In summary, we have studied the switching characteristics of SLAs at transparency current under strong control power. We find that the transparency current is a function of the input control energy due to two photon absorption. By biasing a SLA at transparency current in a loop mirror, an AND gate operation that recovers in 2 ps with maximum nonlinear transmission of $60 \%$ at switching energy of $9 \mathrm{pJ}$ is demonstrated. To evaluate $100 \mathrm{~Gb} / \mathrm{s}$ switching at the transparency condition, we generate a 7-bit $1011001100 \mathrm{~Gb} / \mathrm{s}$ packet as an input control pulse. We find that the transparency conditions are different for the first bit and the remaining bits due to the TPA induced carriers and pump depletion in the SLA. Coupled propagation equations that include TPA, gain saturation from carrier depletion, gain saturation from carrier heating, and gain increase from the TPA are solved numerically. From the simulation results, we verify that the intensity-dependent transparency current is due to the carriers generated by TPA. We also show that the carrier density changed by the strong pump is different at different positions of the SLA because the pump peak power decreases along the propagation in the SLA. Finally, we conclude that to operate the SLA at transparency current for 100 $\mathrm{Gb} / \mathrm{s}$ optical switching, one needs to use either low power to avoid strong TPA or a pulse width that is longer than the SLA transit time.

\section{ACKNOWLEDGMENTS}

This work was sponsored by the NSF and the DOD.

${ }^{1}$ R. J. Manning and D. A. O. Davies, Opt. Lett. 19, 889 (1994).

${ }^{2}$ R. J. Manning and G. Sherlock, Electron. Lett. 31, 307 (1995).

${ }^{3}$ N. S. Patel, K. L. Hall, and K. A. Rauschenbach, Opt. Lett. 21, 1466 (1996). 
${ }^{4}$ K. L. Hall and K. A. Rauschenbach, Optical Fiber Communication Conference, 1998.

${ }^{5}$ K. L. Hall, G. Lenz, A. M. Darwish, and E. P. Ippen, Opt. Commun. 111, 589 (1994)

${ }^{6}$ D. A. O. Davies, M. A. Fisher, D. J. Elton, S. D. Perrin, M. J. Adams, G. T. Kennedy, R. S. Grant, P. D. Roberts, and W. Sibbett, Electron. Lett. 29, 1710 (1993).

${ }^{7}$ B. S. Robinson, and K. L. Hall, Conference on Lasers and Electro-Optics Proceeding, 1998 OSA Technical Digest Series Vol. 6 (Optical Society of America, Washington, DC, 1998), p. 2.

${ }^{8}$ Y.-H. Kao, I. V. Goltser, M. N. Islam, and G. Raybon, Conference on Lasers and Electro-Optics Proceeding, 1997 OSA Technical Digest Series Vol. 11 (Optical Society of America, Washington, DC, 1997), p. 94.

${ }^{9}$ G. T. Kennedy, P. D. Roberts, W. Sibbett, D. A. O. Davies, M. A. Fisher, and M. J. Adams, Conference on Lasers and Electro-Optics Proceeding, 1996 OSA Technical Digest Series Vol. 9 (Optical Society of America,
Washington, DC, 1996), p. 11.

${ }^{10}$ V. Mizrahi, K. W. DeLong, G. I. Stegeman, M. A. Saifi, and M. J. Andrejco, Opt. Lett. 14, 1140 (1989).

${ }^{11}$ K. W. DeLong, and G. I. Stegeman, Appl. Phys. Lett. 57, 2063 (1990).

${ }^{12}$ J. Mork, J. Mark, and C. P. Seltzer, Appl. Phys. Lett. 64, 2206 (1994).

${ }^{13}$ Y.-H. Kao, I. V. Goltser, M. Jiang, M. N. Islam, and G. Raybon, Appl. Phys. Lett. 69, 4221 (1996).

${ }^{14}$ J. M. Tang and K. A. Shore, IEEE J. Quantum Electron. QE-34, 1263 (1998).

${ }^{15}$ M. Y. Hong, Y. H. Chang, A. Dienes, J. P. Heritage, and P. J. Delfyett, IEEE J. Quantum Electron. QE-30, 1122 (1994).

${ }^{16}$ P. J. Delfyett, A. Dienes, J. P. Heritage, M. Y. Hong, and Y. H. Chang, Appl. Phys. B: Lasers Opt. b58, 183 (1994).

${ }^{17}$ G. P. Agrawal and N. A. Olsson, IEEE J. Quantum Electron. QE-25, 2297 (1989). 